\title{
Correction: Abramov, R. The Random Gas of Hard Spheres. J 2019, 2, 162-205
}

\section{Rafail V. Abramovid}

Department of Mathematics, Statistics and Computer Science, University of Illinois at Chicago, 851 S. Morgan St., Chicago, IL 60607, USA; abramov@uic.edu

Received: 9 September 2020; Accepted: 11 September 2020; Published: 16 September 2020

In the published paper [1], we used the spatial correlation function $R(\sigma)$ of two spheres, each of diameter $\sigma$, to construct a closure to the BBGKY hierarchy of hard spheres. In the subsequent derivation of the fluid dynamics equations in [1], we relied upon the assumption that $R(\sigma)=1+O(\sigma)$. We later discovered that $R(\sigma)$ is the two-sphere cavity distribution function for hard spheres [2] in the constant sphere density limit, and thus is not $1+O(\sigma)$. What follows below are the necessary corrections to the relevant equations in [1].

\section{List of Changes}

1. Equation (162) and the following sentence are changed as follows:

$$
F^{(2)}(t, \boldsymbol{x}, \boldsymbol{y}, \boldsymbol{v}, \boldsymbol{w}) \approx e^{-\lambda \Theta_{\alpha \sigma}(\sigma-\|x-y\|)} R^{(2)}((\boldsymbol{x}+\boldsymbol{y}) / 2,\|\boldsymbol{x}-\boldsymbol{y}\|) f(t, \boldsymbol{x}, \boldsymbol{v}) f(t, \boldsymbol{y}, \boldsymbol{w}),
$$

where $R^{(2)}$ depends parametrically on $\alpha$ and $\lambda: R^{(2)}=R_{\lambda, \alpha^{\prime}}^{(2)}$ and, in the case of a spatially nonuniform distribution, is also generally a function of the midpoint between $x$ and $y$.

2. The sentence following Equation (165) is changed as follows:

"We can apply the formula above to our set-up by setting $z_{1}=(\boldsymbol{x}, \boldsymbol{v}), \boldsymbol{z}_{2}=(\boldsymbol{y}, \boldsymbol{w}), F=F^{(2)}$, $\psi=e^{-\lambda \Theta_{\alpha \sigma}(\sigma-\|x-y\|)} R^{(2)}((x+y) / 2,\|x-y\|)$, and $p_{1}=p_{2}=\bar{F}^{(1)}$ from Equation (156)."

3. Equation (167) is changed as follows:

$$
\begin{aligned}
& \frac{\partial f}{\partial t}+\boldsymbol{v} \cdot \frac{\partial f}{\partial x}=(K-1) \lambda \sigma^{2} \int e^{-\lambda \Theta_{\alpha}(1-r)} \delta_{\alpha}(r-1) \boldsymbol{n} \cdot(\boldsymbol{w}-\boldsymbol{v}) \Theta(\boldsymbol{n} \cdot(\boldsymbol{w}-\boldsymbol{v})) \\
& {\left[R_{\lambda, \alpha}^{(2)}(\boldsymbol{x}+\sigma r \boldsymbol{n} / 2, \sigma r) f\left(\boldsymbol{x}, \boldsymbol{v}^{\prime}\right) f\left(\boldsymbol{x}+\sigma r \boldsymbol{n}, \boldsymbol{w}^{\prime}\right)-R_{\lambda, \alpha}^{(2)}(\boldsymbol{x}-\sigma r \boldsymbol{n} / 2, \sigma r) f(\boldsymbol{x}, \boldsymbol{v}) f(\boldsymbol{x}-\sigma r \boldsymbol{n}, \boldsymbol{w})\right] r^{2} \mathrm{~d} r \mathrm{~d} \boldsymbol{n} \mathrm{d} \boldsymbol{w} .}
\end{aligned}
$$

4. The first sentence in Section 6.2 is changed as follows:

"Above in Equation (167), we can formally assume that the contact zone is 'thin' that is, $\alpha \rightarrow 0$ so that, for the values of $r$ for which $\delta_{\alpha}(r-1)>0$, we have $f(x \pm \sigma r n) \rightarrow f(x \pm \sigma n)$, $R_{\lambda, \alpha}^{(2)}(x \pm \sigma r n / 2, \sigma r) \rightarrow R_{\lambda, 0}^{(2)}(x \pm \sigma n / 2, \sigma) .^{\prime \prime}$

5. Equation (169) is changed as follows:

$$
\begin{aligned}
& \frac{\partial f}{\partial t}+\boldsymbol{v} \cdot \frac{\partial f}{\partial x}=(K-1) \sigma^{2}\left(1-e^{-\lambda}\right) \int \boldsymbol{n} \cdot(\boldsymbol{w}-\boldsymbol{v}) \Theta(n \cdot(\boldsymbol{w}-\boldsymbol{v})) \\
& {\left[R_{\lambda, 0}^{(2)}(\boldsymbol{x}+\sigma n / 2, \sigma) f\left(\boldsymbol{x}, \boldsymbol{v}^{\prime}\right) f\left(\boldsymbol{x}+\sigma n, \boldsymbol{w}^{\prime}\right)-R_{\lambda, 0}^{(2)}(\boldsymbol{x}-\sigma n / 2, \sigma) f(\boldsymbol{x}, \boldsymbol{v}) f(\boldsymbol{x}-\sigma \boldsymbol{n}, \boldsymbol{w})\right] \mathrm{d} \boldsymbol{n} \mathrm{d} \boldsymbol{w} .}
\end{aligned}
$$

6. Equation (170) is changed as follows, with the new sentence appended immediately after it:

$$
\begin{aligned}
\frac{\partial f}{\partial t}+\boldsymbol{v} & \cdot \frac{\partial f}{\partial x}=(K-1) \sigma^{2} \int \boldsymbol{n} \cdot(\boldsymbol{w}-\boldsymbol{v}) \Theta(\boldsymbol{n} \cdot(\boldsymbol{w}-\boldsymbol{v})) \\
& {\left[R(\boldsymbol{x}+\sigma n / 2) f\left(\boldsymbol{x}, \boldsymbol{v}^{\prime}\right) f\left(\boldsymbol{x}+\sigma \boldsymbol{n}, \boldsymbol{w}^{\prime}\right)-R(\boldsymbol{x}-\sigma n / 2) f(\boldsymbol{x}, \boldsymbol{v}) f(\boldsymbol{x}-\sigma \boldsymbol{n}, \boldsymbol{w})\right] \mathrm{d} \boldsymbol{n} \mathrm{d} \boldsymbol{w}, }
\end{aligned}
$$


where $R(x)=R_{\infty, 0}^{(2)}(x, \sigma)$ becomes the two-sphere cavity distribution function for hard spheres of diameter $\sigma$.

7. The last sentence before Section 7 is changed as follows:

"This sets $R(\boldsymbol{x} \pm \sigma n / 2)=1, f(\boldsymbol{x} \pm \sigma n)=f(\boldsymbol{x}, \boldsymbol{v}) \ldots$ "

8. Equation (172) is changed as follows:

$$
\begin{aligned}
\frac{\partial g}{\partial t}+\boldsymbol{v} \cdot & \frac{\partial g}{\partial x}=\frac{K-1}{K} \frac{6}{\pi \rho_{s p} \sigma} \int \boldsymbol{n} \cdot(\boldsymbol{w}-\boldsymbol{v}) \Theta(\boldsymbol{n} \cdot(\boldsymbol{w}-\boldsymbol{v})) \\
& {\left[R(\boldsymbol{x}+\sigma \boldsymbol{n} / 2) g\left(\boldsymbol{x}, \boldsymbol{v}^{\prime}\right) g\left(\boldsymbol{x}+\sigma \boldsymbol{n}, \boldsymbol{w}^{\prime}\right)-R(\boldsymbol{x}-\sigma \boldsymbol{n} / 2) g(\boldsymbol{x}, \boldsymbol{v}) g(\boldsymbol{x}-\sigma \boldsymbol{n}, \boldsymbol{w})\right] \mathrm{d} \boldsymbol{n} \mathrm{d} \boldsymbol{w} . }
\end{aligned}
$$

9. Equation (173) is changed as follows:

$$
\begin{aligned}
\frac{\partial g}{\partial t}+\boldsymbol{v} \cdot & \frac{\partial g}{\partial x}=\frac{6}{\pi \rho_{s p} \sigma} \int \boldsymbol{n} \cdot(\boldsymbol{w}-\boldsymbol{v}) \Theta(\boldsymbol{n} \cdot(\boldsymbol{w}-\boldsymbol{v})) \\
& {\left[R(\boldsymbol{x}+\sigma \boldsymbol{n} / 2) g\left(\boldsymbol{x}, \boldsymbol{v}^{\prime}\right) g\left(\boldsymbol{x}+\sigma \boldsymbol{n}, \boldsymbol{w}^{\prime}\right)-R(\boldsymbol{x}-\sigma \boldsymbol{n} / 2) g(\boldsymbol{x}, \boldsymbol{v}) g(\boldsymbol{x}-\sigma \boldsymbol{n}, \boldsymbol{w})\right] \mathrm{d} \boldsymbol{n} \mathrm{d} \boldsymbol{w} . }
\end{aligned}
$$

10. Equation (175), together with the preceding sentence, are changed as follows:

We also note that

$R(\boldsymbol{x} \pm \sigma n / 2) g(\boldsymbol{x}, \boldsymbol{v}) g(\boldsymbol{x} \pm \sigma n, \boldsymbol{w})=R(\boldsymbol{x}) g(\boldsymbol{x}, \boldsymbol{v}) g(\boldsymbol{x}, \boldsymbol{w}) \pm \sigma \sqrt{R(x)} g(\boldsymbol{x}, \boldsymbol{v}) n \cdot \frac{\partial}{\partial x}(\sqrt{R(x)} g(\boldsymbol{x}, \boldsymbol{w}))+\ldots$

11. Equation (180) is changed as follows:

$$
\begin{aligned}
& \frac{\partial g_{0}}{\partial t}+\boldsymbol{v} \cdot \frac{\partial g_{0}}{\partial x}=\frac{6}{\pi \rho_{s p}} \int \boldsymbol{n} \cdot(\boldsymbol{w}-\boldsymbol{v}) \Theta(\boldsymbol{n} \cdot(\boldsymbol{w}-\boldsymbol{v}))\left[R \left(g_{0}\left(\boldsymbol{v}^{\prime}\right) g_{1}\left(\boldsymbol{w}^{\prime}\right)+g_{1}\left(\boldsymbol{v}^{\prime}\right) g_{0}\left(\boldsymbol{w}^{\prime}\right)-\right.\right. \\
& \left.\left.-g_{0}(\boldsymbol{v}) g_{1}(\boldsymbol{w})-g_{1}(\boldsymbol{v}) g_{0}(\boldsymbol{w})\right)+\boldsymbol{n} \cdot \sqrt{R}\left(g_{0}\left(\boldsymbol{v}^{\prime}\right) \frac{\partial\left(\sqrt{R} g_{0}\left(\boldsymbol{w}^{\prime}\right)\right)}{\partial \boldsymbol{x}}+g_{0}(\boldsymbol{v}) \frac{\partial\left(\sqrt{R} g_{0}(\boldsymbol{w})\right)}{\partial \boldsymbol{x}}\right)\right] \mathrm{d} \boldsymbol{n} \mathrm{d} \boldsymbol{w} .
\end{aligned}
$$

12. Equations (185a) and (185b) are changed as follows:

$$
\begin{gathered}
\mathcal{C}[\boldsymbol{v}]=\frac{6}{\pi \rho_{s p}} \int\left(\boldsymbol{v}-\boldsymbol{v}^{\prime}\right) \boldsymbol{n} \cdot(\boldsymbol{w}-\boldsymbol{v}) \Theta(\boldsymbol{n} \cdot(\boldsymbol{w}-\boldsymbol{v})) \boldsymbol{n} \cdot \frac{\partial\left(\sqrt{R} g_{0}(\boldsymbol{w})\right)}{\partial \boldsymbol{x}} \sqrt{R} g_{0}(\boldsymbol{v}) \mathrm{d} \boldsymbol{n} \mathrm{d} \boldsymbol{w} \mathrm{d} \boldsymbol{v}, \\
\mathcal{C}\left[\|\boldsymbol{v}\|^{2}\right]=\frac{6}{\pi \rho_{s p}} \int\left(\|\boldsymbol{v}\|^{2}-\left\|\boldsymbol{v}^{\prime}\right\|^{2}\right) \boldsymbol{n} \cdot(\boldsymbol{w}-\boldsymbol{v}) \Theta(\boldsymbol{n} \cdot(\boldsymbol{w}-\boldsymbol{v})) \boldsymbol{n} \cdot \frac{\partial\left(\sqrt{R} g_{0}(\boldsymbol{w})\right)}{\partial x} \sqrt{R} g_{0}(\boldsymbol{v}) \mathrm{d} \boldsymbol{n} \mathrm{d} \boldsymbol{w} \mathrm{d} \boldsymbol{v} .
\end{gathered}
$$

13. Equations (187a) and (187b) are changed as follows:

$$
\begin{aligned}
& \mathcal{C}[\boldsymbol{v}]=-\frac{6}{\pi \rho_{s p}} \int(\boldsymbol{n} \cdot(\boldsymbol{w}-\boldsymbol{v}))^{2} \Theta(\boldsymbol{n} \cdot(\boldsymbol{w}-\boldsymbol{v})) \boldsymbol{n} \cdot \frac{\partial\left(\sqrt{R} g_{0}(\boldsymbol{w})\right)}{\partial \boldsymbol{x}} \sqrt{R} g_{0}(\boldsymbol{v}) \boldsymbol{n} \mathrm{d} \boldsymbol{n} \mathrm{d} \boldsymbol{w} \mathrm{d} \boldsymbol{v}, \\
& \mathcal{C}\left[\|\boldsymbol{v}\|^{2}\right]=-\frac{6}{\pi \rho_{s p}} \int(\boldsymbol{n} \cdot(\boldsymbol{w}+\boldsymbol{v}))(\boldsymbol{n} \cdot(\boldsymbol{w}-\boldsymbol{v}))^{2} \Theta(\boldsymbol{n} \cdot(\boldsymbol{w}-\boldsymbol{v})) \boldsymbol{n} \cdot \frac{\partial\left(\sqrt{R} g_{0}(\boldsymbol{w})\right)}{\partial x} \sqrt{R} g_{0}(\boldsymbol{v}) \mathrm{d} \boldsymbol{n} \mathrm{d} \boldsymbol{w} \mathrm{d} \boldsymbol{v} .
\end{aligned}
$$

14. Equation (188) is changed as follows:

$$
\mathcal{C}[\boldsymbol{v}]=-\frac{4}{\rho_{s p}} \frac{\partial}{\partial x}\left(R \rho^{2} \theta\right), \quad \mathcal{C}\left[\|\boldsymbol{v}\|^{2}\right]=-\frac{8}{\rho_{s p}} \frac{\partial}{\partial x} \cdot\left(R \rho^{2} \theta \boldsymbol{u}\right) .
$$

15. Equations (189a) and (189b) are changed as follows:

$$
\begin{gathered}
\frac{\partial \rho}{\partial t}+\frac{\partial}{\partial x} \cdot(\rho \boldsymbol{u})=0, \quad \frac{\partial(\rho \boldsymbol{u})}{\partial t}+\frac{\partial}{\partial x} \cdot\left(\rho\left(\boldsymbol{u} \boldsymbol{u}^{T}+\left(1+\frac{4 \rho}{\rho_{s p}} R\right) \theta \boldsymbol{I}\right)\right)=0, \\
\frac{\partial(\rho \epsilon)}{\partial t}+\frac{\partial}{\partial x} \cdot\left(\rho\left(\epsilon+\left(1+\frac{4 \rho}{\rho_{s p}} R\right) \theta\right) \boldsymbol{u}\right)=0 .
\end{gathered}
$$


16. Equations (197a) and (197b) are changed as follows:

$$
\begin{gathered}
\frac{6}{\pi \rho_{s p}} \int(\boldsymbol{n} \cdot(\boldsymbol{w}-\boldsymbol{v}))^{2}\left((\boldsymbol{n} \cdot(\boldsymbol{w}-\boldsymbol{v})) \boldsymbol{n} \boldsymbol{n}^{T}+\boldsymbol{n}(\boldsymbol{v}-\boldsymbol{u})^{T}+(\boldsymbol{v}-\boldsymbol{u}) \boldsymbol{n}^{T}\right) \Theta(\boldsymbol{n} \cdot(\boldsymbol{w}-\boldsymbol{v})) \\
\left(R\left(g_{0}(\boldsymbol{v}) g_{1}(\boldsymbol{w})+g_{1}(\boldsymbol{v}) g_{0}(\boldsymbol{w})\right)-\boldsymbol{n} \cdot \sqrt{R} g_{0}(\boldsymbol{v}) \frac{\partial\left(\sqrt{R} g_{0}(\boldsymbol{w})\right)}{\partial \boldsymbol{x}}\right) \mathrm{d} \boldsymbol{n} \mathrm{d} \boldsymbol{w} \mathrm{d} \boldsymbol{v}= \\
=\rho \theta\left(\frac{\partial \boldsymbol{u}}{\partial \boldsymbol{x}}+\frac{\partial \boldsymbol{u}^{T}}{\partial \boldsymbol{x}}-\frac{2}{3}\left(\frac{\partial}{\partial \boldsymbol{x}} \cdot \boldsymbol{u}\right) \boldsymbol{I}\right)-\frac{8}{3 \rho_{s p}} R \rho^{2} \theta\left(\frac{\partial}{\partial \boldsymbol{x}} \cdot \boldsymbol{u}\right) \boldsymbol{I}, \\
\frac{6}{\pi \rho_{s p}} \int(\boldsymbol{n} \cdot(\boldsymbol{w}-\boldsymbol{v}))^{2}\left[(\boldsymbol{n} \cdot(\boldsymbol{w}-\boldsymbol{v}))^{2} \boldsymbol{n}+(\boldsymbol{n} \cdot(\boldsymbol{w}-\boldsymbol{v}))\left(\boldsymbol{I}+2 \boldsymbol{n} \boldsymbol{n}^{T}\right)(\boldsymbol{v}-\boldsymbol{u})+\right. \\
\left.+\left(\|\boldsymbol{v}-\boldsymbol{u}\|^{2} \boldsymbol{I}+2(\boldsymbol{v}-\boldsymbol{u})(\boldsymbol{v}-\boldsymbol{u})^{T}\right) \boldsymbol{n}\right] \Theta(\boldsymbol{n} \cdot(\boldsymbol{w}-\boldsymbol{v})) \\
\left(R\left(g_{0}(\boldsymbol{v}) g_{1}(\boldsymbol{w})+g_{1}(\boldsymbol{v}) g_{0}(\boldsymbol{w})\right)-\boldsymbol{n} \cdot \sqrt{R} g_{0}(\boldsymbol{v}) \frac{\partial\left(\sqrt{R} g_{0}(\boldsymbol{w})\right)}{\partial \boldsymbol{x}}\right) \mathrm{d} \boldsymbol{n} \mathrm{d} \boldsymbol{w} \mathrm{d} \boldsymbol{v}=5 \rho \theta \frac{\partial \theta}{\partial \boldsymbol{x}}-\frac{20}{\rho_{s p}} \theta \frac{\partial\left(R \rho^{2} \theta\right)}{\partial x} .
\end{gathered}
$$

17. Equations (198a)-(198d) are changed as follows:

$$
\begin{gathered}
\int(\boldsymbol{n} \cdot(\boldsymbol{w}-\boldsymbol{v}))^{2}\left((\boldsymbol{n} \cdot(\boldsymbol{w}-\boldsymbol{v})) \boldsymbol{n} \boldsymbol{n}^{T}+\boldsymbol{n}(\boldsymbol{v}-\boldsymbol{u})^{T}+(\boldsymbol{v}-\boldsymbol{u}) \boldsymbol{n}^{T}\right) \\
\Theta(\boldsymbol{n} \cdot(\boldsymbol{w}-\boldsymbol{v})) R\left(g_{0}(\boldsymbol{v}) g_{1}(\boldsymbol{w})+g_{1}(\boldsymbol{v}) g_{0}(\boldsymbol{w})\right) \mathrm{d} \boldsymbol{n} \mathrm{d} \boldsymbol{w} \mathrm{d} \boldsymbol{v}=-\frac{16 \sqrt{\pi}}{5} R \rho^{2} \sqrt{\theta} \boldsymbol{S}, \\
\int(\boldsymbol{n} \cdot(\boldsymbol{w}-\boldsymbol{v}))^{2}\left((\boldsymbol{n} \cdot(\boldsymbol{w}-\boldsymbol{v})) \boldsymbol{n} \boldsymbol{n}^{T}+\boldsymbol{n}(\boldsymbol{v}-\boldsymbol{u})^{T}+(\boldsymbol{v}-\boldsymbol{u}) \boldsymbol{n}^{T}\right) \\
\Theta(\boldsymbol{n} \cdot(\boldsymbol{w}-\boldsymbol{v})) \boldsymbol{n} \cdot \sqrt{R} g_{0}(\boldsymbol{v}) \frac{\partial\left(\sqrt{R} g_{0}(\boldsymbol{w})\right)}{\partial x} \mathrm{~d} \boldsymbol{n} \mathrm{d} \boldsymbol{w} \mathrm{d} \boldsymbol{v}=\frac{4 \pi}{15} R \rho^{2} \theta\left(\frac{\partial u}{\partial x}+\frac{\partial \boldsymbol{u}}{\partial \boldsymbol{x}}+\left(\frac{\partial}{\partial \boldsymbol{x}} \cdot \boldsymbol{u}\right) \boldsymbol{I}\right), \\
\int(\boldsymbol{n} \cdot(\boldsymbol{w}-\boldsymbol{v}))^{2}\left[(\boldsymbol{n} \cdot(\boldsymbol{w}-\boldsymbol{v}))^{2} \boldsymbol{n}+(\boldsymbol{n} \cdot(\boldsymbol{w}-\boldsymbol{v}))\left(\boldsymbol{I}+2 \boldsymbol{n} \boldsymbol{n}^{T}\right)(\boldsymbol{v}-\boldsymbol{u})+\right. \\
\left.+\left(\|\boldsymbol{v}-\boldsymbol{u}\|^{2} \boldsymbol{I}+2(\boldsymbol{v}-\boldsymbol{u})(\boldsymbol{v}-\boldsymbol{u})^{T}\right) \boldsymbol{n}\right] \Theta(\boldsymbol{n} \cdot(\boldsymbol{w}-\boldsymbol{v})) \\
R\left(g_{0}(\boldsymbol{v}) g_{1}(\boldsymbol{w})+g_{1}(\boldsymbol{v}) g_{0}(\boldsymbol{w})\right) \mathrm{d} \boldsymbol{n} \mathrm{d} \boldsymbol{w} \mathrm{d} \boldsymbol{v}=-\frac{64 \sqrt{\pi}}{15} R \rho^{2} \sqrt{\theta} \boldsymbol{q}, \\
\int(\boldsymbol{n} \cdot(\boldsymbol{w}-\boldsymbol{v}))^{2}\left[(\boldsymbol{n} \cdot(\boldsymbol{w}-\boldsymbol{v}))^{2} \boldsymbol{n}+(\boldsymbol{n} \cdot(\boldsymbol{w}-\boldsymbol{v}))\left(\boldsymbol{I}+2 \boldsymbol{n} \boldsymbol{n}^{T}\right)(\boldsymbol{v}-\boldsymbol{u})+\right. \\
\left.+\left(\|\boldsymbol{v}-\boldsymbol{u}\|^{2} \boldsymbol{I}+2(\boldsymbol{v}-\boldsymbol{u})(\boldsymbol{v}-\boldsymbol{u})^{T}\right) \boldsymbol{n}\right] \Theta(\boldsymbol{n} \cdot(\boldsymbol{w}-\boldsymbol{v})) \\
\boldsymbol{n} \cdot \sqrt{R} g_{0}(\boldsymbol{v}) \frac{\partial\left(\sqrt{R} g_{0}(\boldsymbol{w})\right)}{\partial \boldsymbol{x}} \mathrm{d} \boldsymbol{n} \mathrm{d} \boldsymbol{w} \mathrm{d} \boldsymbol{v}=\frac{10 \pi}{3} \theta \frac{\partial\left(R \rho^{2} \theta\right)}{\partial \boldsymbol{x}}+2 \pi R \rho^{2} \theta \frac{\partial \theta}{\partial \boldsymbol{x}} .
\end{gathered}
$$

18. Equations (199a) and (199b) are changed as follows:

$$
\begin{gathered}
\rho \boldsymbol{S}=\left(\frac{1}{R}+\frac{8 \rho}{5 \rho_{s p}}\right)[\rho \boldsymbol{S}]_{B}=-\left(\frac{1}{R}+\frac{8 \rho}{5 \rho_{s p}}\right) \mu\left(\frac{\partial \boldsymbol{u}}{\partial \boldsymbol{x}}+\frac{\partial \boldsymbol{u}^{T}}{\partial \boldsymbol{x}}-\frac{2}{3}\left(\frac{\partial}{\partial \boldsymbol{x}} \cdot \boldsymbol{u}\right) \boldsymbol{I}\right), \\
\rho \boldsymbol{q}=\left(\frac{1}{R}+\frac{12 \rho}{5 \rho_{s p}}\right)[\rho \boldsymbol{q}]_{B}=-\left(\frac{1}{R}+\frac{12 \rho}{5 \rho_{s p}}\right) \frac{15}{4} \mu \frac{\partial \theta}{\partial x}, \quad \mu=\frac{5 \sqrt{\pi} \rho_{s p} \sigma}{96} \sqrt{\theta},
\end{gathered}
$$


19. Equations (201a) and (201b) are changed as follows:

$$
\begin{aligned}
& \frac{\partial(\rho \boldsymbol{u})}{\partial t}+\frac{\partial}{\partial x} \cdot\left(\rho\left(\boldsymbol{u} \boldsymbol{u}^{T}+\theta \boldsymbol{I}+\sigma \boldsymbol{S}\right)\right)=-\frac{4}{\rho_{s p}} \frac{\partial}{\partial x} \cdot\left(R \rho^{2} \theta\right)+ \\
& +\frac{6 \sigma}{\pi \rho_{s p}} \int(\boldsymbol{n} \cdot(\boldsymbol{w}-\boldsymbol{v}))^{2} \boldsymbol{n} \Theta(\boldsymbol{n} \cdot(\boldsymbol{w}-\boldsymbol{v})) \\
& \sqrt{R}\left(-g_{1}(\boldsymbol{v}) \boldsymbol{n} \cdot \frac{\partial\left(\sqrt{R} g_{0}(\boldsymbol{w})\right)}{\partial x}-g_{0}(\boldsymbol{v}) \boldsymbol{n} \cdot \frac{\partial\left(\sqrt{R} g_{1}(\boldsymbol{w})\right)}{\partial x}+\frac{1}{2} g_{0}(\boldsymbol{v}) \boldsymbol{n}^{T} \frac{\partial^{2}\left(\sqrt{R} g_{0}(\boldsymbol{w})\right)}{\partial \boldsymbol{x}^{2}} \boldsymbol{n}\right) \mathrm{d} \boldsymbol{n} \mathrm{w} \boldsymbol{w} \mathrm{v}, \\
& \frac{\partial(\rho \epsilon)}{\partial t}+\frac{\partial}{\partial x} \cdot(\rho((\epsilon+\theta) \boldsymbol{u}+\sigma \boldsymbol{S} \boldsymbol{u}+\sigma \boldsymbol{q}))=-\frac{4}{\rho_{s p}} \frac{\partial}{\partial x} \cdot\left(R \rho^{2} \theta \boldsymbol{u}\right)+ \\
& +\frac{3 \sigma}{\pi \rho_{s p}} \int(\boldsymbol{n} \cdot(\boldsymbol{w}+\boldsymbol{v}))(\boldsymbol{n} \cdot(\boldsymbol{w}-\boldsymbol{v}))^{2} \Theta(\boldsymbol{n} \cdot(\boldsymbol{w}-\boldsymbol{v})) \\
& \sqrt{R}\left(-g_{1}(\boldsymbol{v}) \boldsymbol{n} \cdot \frac{\partial\left(\sqrt{R} g_{0}(\boldsymbol{w})\right)}{\partial x}-g_{0}(\boldsymbol{v}) \boldsymbol{n} \cdot \frac{\partial\left(\sqrt{R} g_{1}(\boldsymbol{w})\right)}{\partial x}+\frac{1}{2} g_{0}(\boldsymbol{v}) \boldsymbol{n}^{T} \frac{\partial^{2}\left(\sqrt{R} g_{0}(\boldsymbol{w})\right)}{\partial \boldsymbol{x}^{2}} \boldsymbol{n}\right) \mathrm{d} \boldsymbol{n} \mathrm{d} \boldsymbol{w} \mathrm{d} \boldsymbol{v} .
\end{aligned}
$$

20. Equation (202), together with the preceding sentence, are changed as follows:

Above, we take advantage of the fact that, for $\psi(v)=v$ or $\psi(v)=\|v\|^{2}$,

$$
\int\left(\psi\left(\boldsymbol{v}^{\prime}\right)-\psi(\boldsymbol{v})\right) \boldsymbol{n} \cdot(\boldsymbol{w}-\boldsymbol{v}) \Theta(\boldsymbol{n} \cdot(\boldsymbol{w}-\boldsymbol{v}))\left(\boldsymbol{n} \boldsymbol{n}^{T}\right) g_{0}(\boldsymbol{v}) g_{0}(\boldsymbol{w}) \mathrm{d} \boldsymbol{n} \mathrm{d} \boldsymbol{w} \mathrm{d} \boldsymbol{v}=0 .
$$

21. Equations (203a)-(203d) are changed as follows:

$$
\begin{aligned}
& \int(\boldsymbol{n} \cdot(\boldsymbol{w}-\boldsymbol{v}))^{2} n \Theta(\boldsymbol{n} \cdot(\boldsymbol{w}-\boldsymbol{v})) \\
& \sqrt{R}\left(g_{1}(\boldsymbol{v}) \boldsymbol{n} \cdot \frac{\partial\left(\sqrt{R} g_{0}(\boldsymbol{w})\right)}{\partial \boldsymbol{x}}+g_{0}(\boldsymbol{v}) \boldsymbol{n} \cdot \frac{\partial\left(\sqrt{R} g_{1}(\boldsymbol{w})\right)}{\partial \boldsymbol{x}}\right) \mathrm{d} \boldsymbol{n} \mathrm{d} \boldsymbol{w} \mathrm{d} \boldsymbol{v}=\frac{4 \pi}{15} \frac{\partial}{\partial x} \cdot\left(R \rho^{2} \boldsymbol{S}\right), \\
& \int(\boldsymbol{n} \cdot(\boldsymbol{w}-\boldsymbol{v}))^{2} \boldsymbol{n} \Theta(\boldsymbol{n} \cdot(\boldsymbol{w}-\boldsymbol{v})) \boldsymbol{n}^{T} \frac{\partial^{2}\left(\sqrt{R} g_{0}(\boldsymbol{w})\right)}{\partial x^{2}} \boldsymbol{n} \sqrt{R} g_{0}(\boldsymbol{v}) \mathrm{d} \boldsymbol{n} \mathrm{d} \boldsymbol{w} \mathrm{d} \boldsymbol{v}= \\
& =\frac{8 \sqrt{\pi}}{15} \frac{\partial}{\partial x} \cdot\left(R \rho^{2} \sqrt{\theta}\left(\frac{\partial u}{\partial x}+\frac{\partial u^{T}}{\partial x}+\left(\frac{\partial}{\partial x} \cdot u\right) \boldsymbol{l}\right)\right), \\
& \int(\boldsymbol{n} \cdot(\boldsymbol{w}+\boldsymbol{v}))(\boldsymbol{n} \cdot(\boldsymbol{w}-\boldsymbol{v}))^{2} \Theta(\boldsymbol{n} \cdot(\boldsymbol{w}-\boldsymbol{v})) \\
& \sqrt{R}\left(g_{1}(\boldsymbol{v}) \boldsymbol{n} \cdot \frac{\partial\left(\sqrt{\bar{R}} g_{0}(\boldsymbol{w})\right)}{\partial \boldsymbol{x}}+g_{0}(\boldsymbol{v}) \boldsymbol{n} \cdot \frac{\partial\left(\sqrt{\bar{R}} g_{1}(\boldsymbol{w})\right)}{\partial \boldsymbol{x}}\right) \mathrm{d} \boldsymbol{n} \mathrm{d} \boldsymbol{w} \mathrm{d} \boldsymbol{v}=\frac{8 \pi}{15} \frac{\partial}{\partial \boldsymbol{x}} \cdot\left(R \rho^{2}\left(\boldsymbol{S} \boldsymbol{u}+\frac{3}{2} \boldsymbol{q}\right)\right), \\
& \int(\boldsymbol{n} \cdot(\boldsymbol{w}+\boldsymbol{v}))(\boldsymbol{n} \cdot(\boldsymbol{w}-\boldsymbol{v}))^{2} \Theta(\boldsymbol{n} \cdot(\boldsymbol{w}-\boldsymbol{v})) \boldsymbol{n}^{T} \frac{\partial^{2}\left(\sqrt{R} g_{0}(\boldsymbol{w})\right)}{\partial \boldsymbol{x}^{2}} n \sqrt{R} g_{0}(\boldsymbol{v}) \mathrm{d} \boldsymbol{n} \mathrm{d} \boldsymbol{w} \mathrm{d} \boldsymbol{v}= \\
& =\frac{16 \sqrt{\pi}}{15} \frac{\partial}{\partial x} \cdot\left(R \rho^{2} \sqrt{\theta}\left[\left(\frac{\partial u}{\partial x}+\frac{\partial u}{\partial x}^{T}+\left(\frac{\partial}{\partial x} \cdot u\right) \boldsymbol{I}\right) \boldsymbol{u}+\frac{5}{2} \frac{\partial \theta}{\partial x}\right]\right) .
\end{aligned}
$$

22. Equations (204a) and (204b) are changed as follows:

$$
\begin{aligned}
& \frac{\partial(\rho \boldsymbol{u})}{\partial t}+\frac{\partial}{\partial x} \cdot\left(\rho\left(\boldsymbol{u} \boldsymbol{u}^{T}+\theta \boldsymbol{I}\right)\right)=-\frac{4}{\rho_{s p}} \frac{\partial}{\partial x} \cdot\left(R \rho^{2} \theta\right)- \\
& \quad-\sigma \frac{\partial}{\partial x} \cdot\left(\left(1+\frac{8 \rho}{5 \rho_{s p}} R\right) \rho \boldsymbol{S}\right)+\frac{8 \sigma}{5 \sqrt{\pi} \rho_{s p}} \frac{\partial}{\partial x} \cdot\left(R \rho^{2} \sqrt{\theta}\left(\frac{\partial \boldsymbol{u}}{\partial x}+\frac{\partial \boldsymbol{u}^{T}}{\partial x}+\left(\frac{\partial}{\partial \boldsymbol{x}} \cdot \boldsymbol{u}\right) \boldsymbol{I}\right)\right),
\end{aligned}
$$




$$
\begin{aligned}
\frac{\partial(\rho \epsilon)}{\partial t}+\frac{\partial}{\partial x} \cdot(\rho(\epsilon+\theta) \boldsymbol{u})=-\frac{4}{\rho_{s p}} \frac{\partial}{\partial x} \cdot\left(R \rho^{2} \theta \boldsymbol{u}\right)- & \\
- & \sigma \frac{\partial}{\partial x} \cdot\left(\left(1+\frac{8 \rho}{5 \rho_{s p}} R\right) \rho \boldsymbol{S} \boldsymbol{u}\right)-\sigma \frac{\partial}{\partial x} \cdot\left(\left(1+\frac{12 \rho}{5 \rho_{s p}} R\right) \rho \boldsymbol{q}\right)+ \\
& \quad+\frac{8 \sigma}{5 \sqrt{\pi} \rho_{s p}} \frac{\partial}{\partial x} \cdot\left(R \rho^{2} \sqrt{\theta}\left[\left(\frac{\partial \boldsymbol{u}}{\partial x}+\frac{\partial \boldsymbol{u}^{T}}{\partial \boldsymbol{x}}+\left(\frac{\partial}{\partial x} \cdot \boldsymbol{u}\right) \boldsymbol{I}\right) \boldsymbol{u}+\frac{5}{2} \frac{\partial \theta}{\partial x}\right]\right) .
\end{aligned}
$$

23. Equations (205a) and (205b) are changed as follows:

$$
\begin{aligned}
& \frac{\partial(\rho \boldsymbol{u})}{\partial t}+\frac{\partial}{\partial x} \cdot\left(\rho\left(\boldsymbol{u} \boldsymbol{u}^{T}+\left(1+\frac{4 \rho}{\rho_{s p}} R\right) \theta \boldsymbol{I}\right)\right)= \\
& =\frac{\partial}{\partial x} \cdot\left(\mu\left(\left(\frac{1}{R}+a_{1}\right)\left(\frac{\partial u}{\partial x}+\frac{\partial u^{T}}{\partial x}\right)-\frac{2}{3}\left(\frac{1}{R}+a_{2}\right)\left(\frac{\partial}{\partial x} \cdot \boldsymbol{u}\right) \boldsymbol{I}\right)\right), \\
& \frac{\partial(\rho \epsilon)}{\partial t}+\frac{\partial}{\partial x} \cdot\left(\rho\left(\epsilon+\left(1+\frac{4 \rho}{\rho_{s p}} R\right) \theta\right) u\right)=\frac{15}{4} \frac{\partial}{\partial x} \cdot\left(\mu\left(\frac{1}{R}+a_{3}\right) \frac{\partial \theta}{\partial x}\right)+ \\
& +\frac{\partial}{\partial x} \cdot\left(\mu\left(\left(\frac{1}{R}+a_{1}\right)\left(\frac{\partial u}{\partial x}+\frac{\partial u^{T}}{\partial x}\right)-\frac{2}{3}\left(\frac{1}{R}+a_{2}\right)\left(\frac{\partial}{\partial x} \cdot u\right) \boldsymbol{I}\right) \boldsymbol{u}\right) .
\end{aligned}
$$

24. Equations (206a)-(206c) are changed as follows:

$$
\begin{aligned}
& a_{1}\left(\frac{\rho}{\rho_{s p}}\right)=\frac{16 \rho}{5 \rho_{s p}}\left(1+\frac{4 \rho}{5 \rho_{s p}} R\left(1+\frac{12}{\pi}\right)\right), \\
& a_{2}\left(\frac{\rho}{\rho_{s p}}\right)=\frac{16 \rho}{5 \rho_{s p}}\left(1+\frac{4 \rho}{5 \rho_{s p}} R\left(1-\frac{18}{\pi}\right)\right), \\
& a_{3}\left(\frac{\rho}{\rho_{s p}}\right)=\frac{24 \rho}{5 \rho_{s p}}\left(1+\frac{2 \rho}{15 \rho_{s p}} R\left(9+\frac{32}{\pi}\right)\right) .
\end{aligned}
$$

25. The paragraph preceding Equations (207a)-(207c), as well as Equations (207a)-(207c), are removed. In the next paragraph, the reference [2] (which is [46] in the updated manuscript) is provided for cavity distribution functions for hard spheres.

The main results of [1] are unchanged, and the summary remains the same.

Conflicts of Interest: The author declares no conflict of interest.

\section{References}

1. Abramov, R. The Random Gas of Hard Spheres. J 2019, 2, 162-205. [CrossRef]

2. Boublík, T. Hard-Sphere Radial Distribution Function from the Residual Chemical Potential. Molec. Phys. 2006, 104, 3425-3433. [CrossRef]

(C) 2020 by the author. Licensee MDPI, Basel, Switzerland. This article is an open access article distributed under the terms and conditions of the Creative Commons Attribution (CC BY) license (http://creativecommons.org/licenses/by/4.0/). 